\title{
Literacia em saúde: um retrato da população adulta portuguesa
}

\section{Health literacy: a portrait of the portuguese adults population}

\author{
Vânia Carneiro*, Isabel Silva**, Glória Jólluskin** \\ *UFP, ** UFP/Centro de Investigação FP-B2S/APASD
}

\begin{abstract}
Resumo
O presente estudo tem como objetivo descrever os níveis de literacia em saúde em adultos portugueses. Foi estudada uma amostra de conveniência constituída por 316 indivíduos, com idades compreendidas entre os 18 e os 78 anos $(M=35.12 ; D P=14.49), 66.1 \%$ do sexo feminino. Os participantes responderam a um questionário sociodemográfico e à Escala de Literacia em Saúde. Os resultados indicam que os níveis globais de Literacia em Saúde são medianos. Ao nível da Literacia Comunicacional, os participantes revelam possuir competências razoáveis, porém ao nível da Literacia Funcional estas são apenas medianas e ao nível da Literacia Crítica são claramente fracas.

Palavras-chave: literacia, saúde, avaliação, questionário
\end{abstract}

\begin{abstract}
The present study aims to describe the level of health literacy in Portuguese adults. A cohort of 316 individuals, aged between $18-78$ years $(M=35.12 ; S D=14.49)$, was studied, $66.1 \%$ female. Participants answered to a sociodemographic questionnaire and to the Health Literacy Scale. Results indicate that the overall levels of Health Literacy are medium. At the level of the Communication Literacy, participants reveal to possess reasonable skills, however in the Functional Literacy these are only median and, at the level of Critical Literacy, are clearly weak.

Keywords: literacy, health, evaluation, questionnaire
\end{abstract}

A definição do conceito "literacia em saúde" nem sempre foi consensual, no entanto, foi a partir da década que 90, que esta ganhou maior consistência e importância, passando a estar vinculada às questões relacionadas com a educação e promoção para a saúde (Nutbeam, 2000).

Segundo Carmo (2016, p.42), a literacia em saúde: "implica que o indivíduo seja capaz de, designadamente, descrever e comunicar sintomas fisicos e mentais, compreender as orientações dos profissionais, tomar decisões acerca de tratamentos médicos e do quando e como procurar ajuda médica, compreender instruções e prescrições, folhetos médicos, de consentir de forma livre e esclarecida e, ainda, seja capaz de negociar com outras entidades financiadoras".

Assim, compreende-se que, quando nos referimos a este construto, estamos perante um reflexo que espelha um papel ativo, dinâmico e crítico por parte dos indivíduos, aos mais variados níveis (pessoal, social e profissional) como utente, consumidor e cidadão (Carmo, 2016), uma vez que, não só exige que o utente saiba frequentar o sistema de saúde, como também exige enquanto consumidor que o indivíduo seja consciente e autónomo nas suas escolhas e, enquanto cidadão exige que este se integre em organizações e participe confortavelmente de livre e espontânea vontade em debates de saúde, por exemplo, sem esquecer quais são os seus direitos e deveres, adotando, deste modo, comportamentos informados relativos à mesma, tal como é mencionado no portal do Serviço Nacional de Saúde (2010).

Neste sentido, Nutbeam (2000) refere que, ao falar de literacia em saúde, estamos inevitavelmente a possibilitar que esta seja perspetivada como estando em crescente autonomia e como devendo ser alvo da capacitação (empowerment) dos indivíduos, uma vez que, este afirma e defende a existência de três grandes tipos ou níveis de literacia em saúde - a funcional e/ou básica, a interativa e/ou comunicacional e por fim, a crítica.

Segundo o mesmo autor, a literacia em saúde básica e/ou funcional ilustra competências como o saber ler, escrever e calcular, permitindo um adequado funcionamento no contexto diário e integração nos contextos de saúde; a literacia em saúde interativa e/ou comunicacional resulta da interação entre capacidades cognitivas e sociais, que são usadas diariamente para extrair informações, conhecimentos e significados, de modo a, posteriormente, dar uso a essa recolha através da participação ativa nos cuidados de saúde e, por último, a literacia em saúde crítica, que, tal como na anterior, integra competências cognitivas e sociais que podem e devem ser usadas para refletir criticamente informações adquiridas, com o intuito de posteriormente usar essas mesmas informações para adotar comportamentos de controlo, tomada de posição e poder face aos acontecimentos que vão surgindo ao longo do ciclo vital, nomeadamente através da participação em ações e tomadas de decisões ligadas à saúde e doença (Nutbeam, 2000).

No que diz respeito à literacia em Portugal, o estudo desenvolvido por Benavente, et al. (1996), intitulado de "A literacia em Portugal: resultados de uma pesquisa extensiva e monográfica", chegou à conclusão de que, nos anos 90, o perfil dominante de literacia da população portuguesa se enquadra no nível 1 em todas as competências - leitura, escrita e cálculo. É de referir, que 
este nível é dos menos exigentes. Implica que as pessoas através de textos simples e pequenos identifiquem palavras, façam a transcrição literal e/ou executem um cálculo aritmético simples.

Em 2012, o projeto Saúde que Conta desenvolveu em Portugal o primeiro estudo sobre esta temática no nosso país, com o objetivo de aferir de que forma a literacia em saúde influencia três domínicos fundamentais da gestão nesta temática: a) resultados em saúde; b) a utilização dos serviços de saúde e c) a otimização dos gastos em saúde. Assim, surgiram as primeiras conclusões sobre os níveis de literacia em saúde e a sua relação com e nos estados de saúde dos indivíduos portugueses. As conclusões apontam que, a maioria dos portugueses inquiridos tem um nível de literacia em saúde problemático ou inadequado. Segundo o grupo de peritos estes resultados influenciam negativamente os resultados em saúde, a utilização dos serviços e os gastos em saúde, que são excessivos. Acrescentam ainda, que tudo isto se reflete através de: maior predisposição para adoção de comportamentos de risco e prejudiciais para a saúde, o que conduz inevitavelmente a piores condições de saúde, maior taxa de morbilidade face a certas doenças crónicas, menor utilização de cuidados preventivos e paralelamente, maior taxas de hospitalizações e maior recurso a urgências médicas (Saúde que Conta, 2012).

Passados dois anos, em 2014, foi administrado em Portugal o Questionário Europeu de Literacia em Saúde (HLS-EU), que teve como principal objetivo fazer um retrato do país nesta matéria e estabelecer comparações com outros países (Espanha, Irlanda, Holanda, Alemanha, Áustria, Grécia, Polónia e Bulgária) quanto ao nível de compreensão que os portugueses tem face às questões relacionadas com a saúde e prevenção da doença. Os resultados demonstram que, mais de metade dos inquiridos portugueses, mais concretamente, $61.1 \%$ apresenta um nível de literacia geral em saúde problemático, sendo o segundo país com pior resultado, ficando atrás da Bulgária. Ao nível da promoção da saúde, $60.2 \%$ apresenta um nível de literacia problemático ou inadequado; ao nível dos cuidados de saúde apenas $44.2 \%$ apresenta um nível suficiente ou excelente de literacia em saúde, e por último, ao nível da prevenção da doença, apenas $45 \%$ dos inquiridos afirma ter um nível suficiente ou excelente de literacia em saúde (Pedro, et al. 2016; Saúde que Conta, 2014). Assim, é na dimensão dos cuidados de saúde que os resultados parecem ser mais alarmantes.

É de salientar, também que $56.2 \%$ dos inquiridos não sabem diferenciar se a informação disponibilizada pelos meios de comunicação sobre saúde é ou não de confiança e que $55 \%$ não sabe de que forma as políticas podem afetar a sua saúde (Saúde que Conta, 2014).

Um estudo recente desenvolvido pela Entidade Reguladora de Saúde (ERS, 2017), que teve como principais objetivos avaliar o nível de literacia dos cidadãos sobre os seus direitos enquanto utentes dos serviços de saúde, bem como avaliar a literacia dos profissionais de saúde e administrativos no que respeita aos direitos dos utentes adultos, chegaram à conclusão que, a maioria dos utentes apresenta um índice global de conhecimento inadequado. Os temas em que os utentes revelam um nível de conhecimento mais deficitário foram: o consentimento informado, os Tempos Máximos de Resposta Garantidos (TMRG) para a realização de consultas nos cuidados primários e nos hospitais e para a realização de cirurgias e o Programa Nacional de Promoção da Saúde Oral (ERS, 2017).

Em particular, desconhecem que o consentimento informado é sempre necessário e que não tem de ser realizado por escrito. Existe também um elevado desconhecimento sobre alguns Meios Complementares de Diagnóstico e Terapêutica (MCDT) e do conceito de testamento vital. Segundo o mesmo estudo, a grande maioria dos utentes e profissionais desconhece, ainda, os grupos de beneficiários do cheque-dentista. Constatou-se que o nível de literacia dos profissionais é maioritariamente inadequado, uma vez que menos de metade dos profissionais de saúde e administrativos revelaram ter um conhecimento suficiente sobre os direitos dos utentes (ERS, 2017).

No que diz respeito a variáveis sociodemográficas e literacia em saúde, os estudos desenvolvidos em Portugal apontam para as seguintes conclusões: a literacia em saúde tende a ser menor no sexo feminino, comparativamente ao sexo masculino (Cavaco \& Santos, 2012; Cunha et al. 2014; Serrão, et al. 2015; Veiga \& Serrão, 2016). Com a idade, o que se verifica é uma correlação negativa, o que indica que as duas variáveis tendem a oscilar no sentido inverso, quanto maior a idade, menor os níveis de literacia (Cunha et al. 2014; Espanha \& Ávila, 2016; Pedro et al. 2016; Veiga \& Serrão, 2016). Contudo, no estudo desenvolvido por Cavaco e Santos (2012) não se encontrou associações entre a literacia em saúde e os diferentes grupos etários. Relativamente à escolaridade, os estudos apontam que, quanto maior for o nível de escolaridade, maiores os níveis de literacia em saúde (Cavaco \& Santos, 2012; Espanha \& Ávila, 2016; Pedro et al. 2016 Serrão, et al. 2015; Veiga \& Serrão, 2016), referem ainda, que são os casados quem apresentam valores superiores de literacia em saúde, quando comparados com os viúvos (Serrão et al. 2015; Veiga \& Serrão, 2016) e os indivíduos ativos (Cavaco \& Silva, 2012; Pedro et al. 2016). Indivíduos com estatuto social mais baixo (Cunha et al. 2014) e com diagnóstico de algum tipo de doença (Espanha \& Ávila, 2016; Veiga \& Serrão, 2016) também revelam piores resultados face à literacia em saúde. Para terminar, estudos apontam ainda, a existência de uma relação positiva e fraca entre a literacia em saúde e qualidade de vida, indicando, assim, que quanto maior for a perceção da qualidade de vida, maior é a literacia em saúde (Espanha \& Ávila, 2016; Veiga \& Serrão, 2016).

Deste modo, compreende-se que aumentar a literacia em saúde nas comunidades têm sido, nas últimas décadas, uma preocupação regular na definição de políticas de saúde, contemplada inclusivamente no atual Plano Nacional de Saúde (2016). No entanto, apesar desta consciência política estar presente nos últimos tempos, o fato é que existem poucos estudos em Portugal que deem uma perspetiva holística sobre o conhecimento que a população portuguesa tem relativamente à saúde, uma vez que, grande parte dos estudos existentes no nosso país sobre este domínio, são demasiado específicos 
em termos da temática/área de saúde em estudo ou então são locais/regionais (Santos, 2010).

Assim, o presente estudo assenta num estudo descritivo, exploratório transversal que tem como principal objetivo descrever os níveis de literacia em saúde (literacia em saúde básica/funcional, literacia em saúde comunicacional/interativa e literacia em saúde crítica), numa amostra adulta portuguesa tendo em consideração algumas das características sociodemográficas dos participantes.

\section{Método}

\section{Participantes}

Foi estudada uma amostra de conveniência constituída por 316 indivíduos portugueses, com idades compreendidas entre os 18 e os 78 anos $(M=35.12$; $D P=14.49)$, dos quais $66.1 \%$ são do sexo feminino e os restantes $(33.9 \%)$ do sexo masculino. A maioria dos participantes tem um nível de escolaridade correspondente ao Ensino Superior (63.9\%), dos quais $31.6 \%$ são licenciados, $20.3 \%$ são mestres e $12 \%$ doutorados, sendo que $5.1 \%$ frequentou apenas o $1^{\circ}$ Ciclo do Ensino Básico/Escola Primária ( $1^{\mathrm{a}}$ à $4^{\mathrm{a}}$ classe), 3.8\% o $2^{\circ}$ Ciclo do Ensino Básico/Ensino Preparatório $\left(5^{\circ}\right.$ e $6^{\circ}$ ano), $7.6 \%$ o $3^{\circ}$ Ciclo do Ensino Básico (do $7^{\circ}$ ao $9^{\circ}$ ano) e por último, $19.3 \%$ concluiu o Ensino Secundário. 18\% refere sofrer alguma doença, sendo que, 14 destes participantes são do sexo masculino e 43 do sexo feminino. A frequência da existência de diagnóstico de uma doença é igual para os dois sexos $\left(\chi^{2}=2.75 ; p>.05\right)$, e os participantes que referem sofrer de alguma doença não diferem dos restantes quanto à idade $(t=1.80 ; p>.05)$. Porém, há uma maior percentagem de participantes do sexo feminino com uma escolaridade ao nível dos três ciclos de estudos do ensino superior $\left(\chi^{2}=2.38 ; \mathrm{p}<.001\right)$.

\section{Instrumentos}

Os participantes responderam a dois instrumentos. Primeiramente ao questionário sociodemográfico e, de seguida, à Escala de Literacia em Saúde (ELS - Silva, Jólluskin \& Carneiro, versão em estudo):

- Questionário sociodemográfico: o questionário sociodemográfico utilizado foi desenvolvido especificamente para o presente estudo e teve como objetivo recolher dados sociodemográficos dos participantes, nomeadamente: sexo, idade, escolaridade e se sofre de alguma doença.

- Escala de Literacia em Saúde - ELS (Silva, Jólluskin \& Carneiro, versão em estudo) é composta por 111 itens totais, que visam avaliar três dimensões fundamentais da literacia em saúde: a literacia funcional, comunicacional e crítica. A dimensão da literacia funcional engloba 21 itens, que se referem ao acesso à informação sobre saúde. A literacia comunicacional integra 31 itens, que se referem à vertente da compreensão e comunicação, isto é, à capacidade para compreender e comunicar informação relacionada com a saúde. Por último, a literacia crítica incluí 59 itens relacionados com a reflexão e tomada de decisão, ou seja, com a capacidade de usar de forma crítica e reflexiva a informação sobre saúde.
Para cada um dos itens constituintes da escala, são oferecidas ao participante cinco opções de resposta (escala de Likert), ora de facilidade/dificuldade ("muito dificil; dificil; nem dificil nem fácil; fácil; muito fácil;") ora de concordância/discordância ("sempre, muitas vezes; algumas vezes; raramente; nunca") relativamente ao comportamento ou situação apresentado/a. O cálculo do score total e de cada subescala é feito através da soma dos valores obtidos em cada item, sendo esse somatório final convertido em percentagem. Assim, o valor mínimo que é possível obter é 0 e o máximo é 100 , sendo que quanto maior o valor, maior é o nível de literacia em saúde apresentado pelo participante. No presente estudo, a ELS revelou apresentar uma elevada fidelidade para a escala global $(\alpha=.97)$, bem como para as três respetivas subescalas: literacia em saúde funcional $(\alpha=.87)$, literacia em saúde comunicacional $(\alpha=.97)$ e literacia em saúde crítica $(\alpha=.96)$.

\section{Procedimento}

Foi solicitada a autorização à Comissão de Ética da Universidade Fernando Pessoa para realização do presente estudo, tendo sido obtido parecer positivo. Após parecer positivo, foi realizado um convite à participação através de redes sociais e mailing lists institucionais, tendo sido os questionários administrados através de viaonline, mais concretamente, através do formulário criado no Google Docs. A recolha decorreu entre os meses de Fevereiro e Abril de 2017, após autorização livre e concedida pelos participantes.

Os dados recolhidos foram instantaneamente codificados numa base de dados em Excel e, posteriormente, transferidos para o Statistical Package for Social Sciences (SPSS) com o intuito de se proceder à análise estatística dos mesmos.

A seleção de participantes obedeceu a quatro requisitos essenciais: (a) serem maiores de idade, ou seja, indivíduos com idade igual ou superior a 18 ano; (b) de língua materna portuguesa; (c) sem perturbações do estado de consciência e (d) capazes de dar o seu consentimento informado de forma livre.

\section{Resultados}

Os resultados indicam que, globalmente, os níveis de literacia em saúde apresentados são medianos $(M=57.37$; $D P=10.03$ ). Ao nível das três subescalas a pontuação mais alta é da Literacia Comunicacional, que apresenta valores razoáveis $(M=73.87 ; D P=15.56)$, seguida da Literacia Funcional $(M=63.69 ; D P=13.30)$, e por último, a Crítica, que se apresenta como baixa $(M=31.99$; $D P=7.03$ ).

A análise dos dados revela, ainda, que, quando consideramos o sexo, não existem diferenças estatisticamente significativas relativamente à literacia em saúde geral $(t=1.11 ; p>.05)$, literacia em saúde funcional $(t=.99 ; p>.05)$, comunicacional $(t=1,35$; $p>0,05)$ e crítica $(t=.99 ; p>.05)$.

Verificou-se que existe uma correlação estatisticamente significativa, negativa e fraca entre a idade e a literacia em saúde geral $(r=.31 ; p>.01)$, assim como, entre aquela e as subescalas literacia em saúde funcional $(r=.39 ; p<.0001)$ e literacia em saúde crítica 
$(r=.21 ; p<.01)$. Porém, verificou-se não existir uma correlação estatisticamente significativa entre a idade e a literacia em saúde comunicacional ( $p>.05)$.

No que diz respeito à escolaridade, verificou-se que são os indivíduos com frequência de apenas o Ensino Básico aqueles que apresentam níveis mais baixos de literacia em saúde em todos os domínios: literacia em saúde geral, $F=3.78 ; \quad p<.01, \quad$ funcional, $\quad F=11.32 ; \quad p<.0001$, comunicacional, $F=4.05 ; p<.001$ e crítica, $F=5.74$; $p<.0001$.

Tabela 1.

Niveis de escolaridade e resultados na ELS (total e por domínios)

\begin{tabular}{|c|c|c|c|c|}
\hline $\begin{array}{c}\text { Nível de } \\
\text { Escolaridade }\end{array}$ & $\begin{array}{c}\text { Literacia } \\
\text { Total } \\
M \\
D P \\
\end{array}$ & $\begin{array}{c}\text { Literacia } \\
\text { Funcional } \\
M \\
D P \\
\end{array}$ & $\begin{array}{c}\text { Literacia } \\
\text { Comuni. } \\
M \\
D P \\
\end{array}$ & $\begin{array}{c}\text { Literacia } \\
\text { Crítica } \\
M \\
D P \\
\end{array}$ \\
\hline $1^{\circ}$ Ciclo do & 33.31 & 42.68 & 60.78 & 20.85 \\
\hline $\begin{array}{l}\text { Ensino } \\
\text { Básico }\end{array}$ & 15.12 & 11.44 & 17.77 & 4.20 \\
\hline $2^{\circ}$ Ciclo do & 44.44 & 41.07 & 61.17 & 25.09 \\
\hline $\begin{array}{l}\text { Ensino } \\
\text { Básico }\end{array}$ & 10.86 & 6.84 & 19.65 & 7.93 \\
\hline $3^{\circ}$ Ciclo do & 55.77 & 56.05 & 69.20 & 28.75 \\
\hline $\begin{array}{l}\text { Ensino } \\
\text { Básico }\end{array}$ & 8.97 & 14.89 & 16.69 & 5.72 \\
\hline Ensino & 55.63 & 61.97 & 70.84 & 31.02 \\
\hline Secundário & 8.45 & 11.22 & 12.38 & 5.43 \\
\hline $1^{\circ}$ Ciclo do & 58.17 & 66.20 & 70.02 & 31.87 \\
\hline $\begin{array}{l}\text { Ensino } \\
\text { Superior }\end{array}$ & 9.22 & 10.44 & 14.87 & 7.09 \\
\hline $2^{\circ}$ Ciclo do & 59.70 & 67.98 & 80.20 & 34.63 \\
\hline $\begin{array}{l}\text { Ensino } \\
\text { Superior }\end{array}$ & 9.24 & 12.60 & 14.69 & 5.86 \\
\hline $3^{\circ}$ Ciclo do & 59.89 & 70.50 & 79.16 & 34.67 \\
\hline $\begin{array}{l}\text { Ensino } \\
\text { Superior }\end{array}$ & 57.36 & 10.79 & 15.69 & 8.73 \\
\hline
\end{tabular}

Legenda: Literacia Comuni. - Literacia Comunicacional

Assumindo-se que, à partida, quanto maior a idade da população portuguesa, menor será a sua escolaridade (Pordata, 2017), procedeu-se a uma análise das correlações parciais entre a idade e a literacia em saúde, controlando-se o nível de escolaridade dos participantes. Os resultados revelam que, ao controlamos esta variável, as associações entre idade e literacia em saúde globalmente considerada, literacia em saúde funcional, literacia em saúde comunicacional e literacia em saúde crítica deixam de ser estatisticamente significativas $(p>.05)$.

\section{Discussão}

Após a descrição dos resultados, torna-se fundamental fazer o contraponto entre os mesmos e o estado de arte relativamente à temática aqui abordada.

Os resultados indicam que, os níveis gerais de literacia em saúde da amostra estudada são medianos, o que não vai de encontro aos estudos já existentes, uma vez que estes afirmam que o nível de literacia em saúde geral em
Portugal é problemático ou inadequado (Cunha, et al. 2014; Pedro, et al. 2016; Veiga \& Serrão, 2016).

O fato de na Escala de Literacia em Saúde - ELS, a dimensão da literacia crítica ter sido a que teve pontuação mais baixa, revela que os indivíduos inquiridos têm capacidades escassas ao nível da reflexão e tomada de decisão face a questões relacionadas com saúde. Tendo em consideração a definição de Nutbeam (2000), podese constatar que os indivíduos inquiridos no presente estudo não se sentem confiantes para adotar comportamentos de controlo, tomada de posição e poder face aos acontecimentos que vão surgindo ao longo do ciclo vital, nomeadamente através da participação em ações e tomadas de decisões ligadas à saúde e doença.

Relativamente ao sexo e literacia em saúde, não foram encontradas diferenças estatisticamente significativas. Os resultados não vão de encontro aos apresentados na literatura, uma vez que estes referem que a literacia em saúde tende a ser menor no sexo feminino, comparativamente ao sexo masculino (Cavaco \& Santos, 2012; Cunha et al. 2014; Serrão, et al. 2015; Veiga \& Serrão, 2016).

No que diz respeito à idade, verificou-se que à medida que a idade aumenta, o nível de literacia em saúde diminuiu, tal como foi constatado nos estudos já referidos (Cunha et al. 2014; Espanha \& Ávila, 2016; Pedro et al. 2016; Veiga \& Serrão, 2016) com a exceção de um (Cavaco \& Santos, 2012).

Para além disso, na variável escolaridade, os resultados sugerem que quanto maior o nível de escolaridade, maior o nível de literacia em saúde. Estes resultados são corroborados com os apresentados na literatura (Cavaco \& Santos, 2012; Espanha \& Ávila, 2016; Pedro et al. 2016 Serrão, et al. 2015; Veiga \& Serrão, 2016). No entanto, quando controlada a variável da escolaridade, as diferenças entre a idade e literacia em saúde, desaparecem. Este dado, indica e confirma que a escolaridade é uma variável que apresenta um papel muito determinante nos níveis médios de literacia em saúde (Cavaco \& Santos, 2012; Espanha \& Ávila, 2016; Pedro, et al. 2016; Serrão, et al. 2015; Veiga \& Serrão, 2016).

Por outro lado, não se verificou existirem diferenças estatisticamente significativas entre os indivíduos que sofrem de alguma doença e entre aqueles que não sofrem de doença, face aos níveis de literacia em saúde, o que não vai de encontro à literatura, uma vez que estes defendem que indivíduos com algum diagnóstico médico tendem a ter valores inferiores de literacia em saúde (Espanha \& Ávila, 2016; Veiga \& Serrão, 2016).

Os resultados apontam ainda, para a não existência de diferenças estatisticamente significativas entre os indivíduos que têm e os que não têm doença, quanto à literacia em saúde globalmente considerada, literacia em saúde funcional, literacia em saúde comunicacional e literacia em saúde crítica.

Assim, tendo em conta este retrato, entende-se que é fundamental a elaboração e a implementação de programas de educação para a saúde junto desta população. Porém, uma vez que os níveis de literacia em saúde na população em geral são medianos, considera-se que este tipo de intervenções poderia ser igualmente 
estendido a todos os cidadãos, sem nunca esquecer que a literacia em saúde não é um problema individual, nem do individuo em singular, mas sim, um determinante sistémico, que deve ser olhado através de uma visão holística. Com base nos resultados apresentados, esta sugestão de programas para a população em geral não necessita de fazer distinção entre os sexos, nem idades, mas sim, ter em consideração o nível de escolaridade e diante de todas as literacias em saúde aqui abordadas, dar especial atenção para a literacia em saúde crítica, sem nunca esquecer as restantes (literacia em saúde funcional e literacia em saúde comunicacional).

Ao elaborar programas de intervenção com o propósito de implementar ações relacionadas com a promoção de literacia em saúde, deve-se dar especial atenção não somente a questões diretamente relacionadas com a saúde, mas também com as condições de vida, sinais sociais, demográficos e económicos, uma vez que, estes são indicadores que influenciam fortemente os níveis de literacia em saúde (Pedro, et al. 2016). Segundo Pedro et al. (2016) é fundamental a existência de uma estratégia nacional de literacia em saúde que inclua a literacia em saúde no próprio sistema nacional de saúde, que a integre no sistema educativo, que assegure a comunicação efetiva entre utente-profissional, bem como o acesso e gestão de informação eficiente e por último, que garanta a sustentabilidade nas iniciativas de promoção de literacia em saúde.

\section{Referências}

Benavente, A., Rosa., A., Costa, A., \& Ávila, P. (1996). A literacia em Portugal. Resultados de uma pesquisa extensiva e monográfica. Lisboa: Fundação Calouste Gulbenkian.http://www.cnedu.pt/pt/publicacoes/estud os-e-relatorios/outros/799-a-literacia-em-portugalresultados-de-uma-pesquisa-extensiva-e-monografica

Carmo, M. (2016). A (i)literacia em saúde: Forças e oportunidades do sistema de saúde português. Gestão Hospitalar,

42-45. https://issuu.com/apah/docs/gh8_jan2016_web

Cavaco, A., \& Santos, A. (2012). Avaliação da legibilidade de folhetos informativos e literacia em saúde. Revista Saúde Pública, 46, 918-922. http://www.scielo.br/scielo.php?script=sci_arttext\&pi $\mathrm{d}=$ S0034-89102012000500019

Cunha, M., Gaspar, R., Fonseca, S., Almeida, D., Silva, M., \& Nunes, L. (2014). Implications of literacy for health for body mas índex. Atención Primária, 46, 180186.http://www.sciencedirect.com/science/article/pii/ S0212656714700885

Entidade Reguladora de Saúde (2017). Literacia em direitos dos utentes de cuidados de saúde. Retrieved https://www.ers.pt/pages/73?news_id=1557

Espanha, R., \& Ávila, P. (2016). Health literacy survey Portugal: A contribution for the knowledge on health and communications. Procedia Computer Science, 100, 1033-1041. http://www.sciencedirect.com/science/article/pii/S187 7050916324462

Nutbeam, D. (2000). Health Literacy as a Public Health Goal: A challenge for contemporary health education and communication strategies into the 21 st century. Health Promotion International, 15, 259-267. https://academic.oup.com/heapro/article/15/3/259/551 108/Health-literacy-as-a-public-health-goal-a

Pedro, A., Amaral, O., \& Escoval, A. (2016). Literacia em saúde, dos dados à ação: Tradução, validação e aplicação do European Health Literacy Survey em Portugal. Revista Portuguesa de Saúde Pública, 34, 259-275.http://www.elsevier.pt/pt/revistas/revistaportuguesa-saude-publica 323/pdf/S0870902516300311/S200/

Portada (2017). Base de Dados de Portugal Contemporâneo. Retrieved http://www.pordata.pt/.

Santos, O. (2010). O papel da literacia em saúde: Capacitando a pessoa com excesso de peso para o controlo e redução da carga ponderal. Endocrinologia, Diabetes \& Obesidade, 4, 127-134. https://dspace.uevora.pt/rdpc/handle/10174/2320?loca le $=p t$

Saúde que Conta (2014). Aplicação em Portugal do questionário europeu de literacia em saúde. Retrieved http://www.saudequeconta.org/investigacao/20132014/.

Saúde que Conta (2012). Think Tank literacia em saúde. Retrievedhttp://www.saudequeconta.org/investigacao/ 20112012-2/.

Serrão, C. (2014). Manual de boas práticas. Porto: Edição Projeto Literacia Saúde. http://recipp.ipp.pt/bitstream/10400.22/5979/1/Manua $1 \% 20$ de $\% 20$ Boas $\% 20 \operatorname{Pr} \% \mathrm{C} 3 \%$ A 1 ticas $\% 20$ $\% 20$ Literacia $\% 20$ em $\% 20$ sa $\%$ C3 $\%$ BAde $\% 20$ na $\% 20$ te rceira\%20idade.pdf

Serrão, C., Veiga, S., \& Vieira, I. (2015). Literacia em saúde: Resultados obtidos a partir de uma amostra de pessoas idosas portuguesas. Revista Portuguesa de Enfermagem de Saúde Mental, 2, 33-38. http://www.scielo.mec.pt/scielo.php?script=sci_arttex t\&pid=S1647-21602015000100006

Serviço Nacional de Saúde (2010). Literacia em saúde. Retrieved

http://www2.insa.pt/sites/INSA/Portugues/AreasCient ificas/PSDC/AreasTrabalho/LiteraciaSaude/Paginas/i nicial.aspx

Veiga, S., \& Serrão, C. (2016). Health literacy of a sample of portuguese elderly. Applied Research in Health And Social Sciences: Interface And Interaction, 13 , 14-26. https://www.degruyter.com/view/j/arhss.2016.13.issu e-1/arhss-2016-0003/arhss-2016-0003.xml 